\title{
Síndrome de Horner durante anestesia neuroaxial en anestesia obstétrica - revisión de la literatura
}

\author{
Horner syndrome during neuraxial anesthesia in obstetric \\ anesthesia - literature review
}

Anamaria Leguizamón Stevenson ${ }^{1,2}$

\begin{abstract}
Background: Obstetric neuraxial block is often used during labor; however, as in all medical procedures, complications have been reported, among which is Horner's syndrome. Objective: Characterize the incidence, pathophysiology and management of Horner's syndrome during neuraxial anesthesia. Methods: A review of literature was conducted by searching articles related to the subject in PubMed and Web of Science. Results: The incidence of Horner's syndrome after neuraxial block is very variable being higher in cases of cesarean section than during labor ( $4 \%$ vs $0.4 \%-1.3 \%$ ). Factors that promote cephalic spread of the anesthetic, increased neuronal sensitivity, physiological and anatomical changes induced by pregnancy, and in some cases poor positioning of the catheter, explain this association. Most of the time it is a benign phenomenon which resolves spontaneously in less than 4 hours; nevertheless, the onset of hemodynamic instability or atypical symptoms must be monitored, which obliges to expand the diagnostic studies and/or interrupt the anesthetic.
\end{abstract}

\section{RESUMEN}

Antecedentes: El bloqueo neuroaxial obstétrico se emplea frecuentemente durante el trabajo de parto; sin embargo, como en todo procedimiento se han reportado complicaciones, dentro de las cuales se encuentra el síndrome de Horner. Objetivo: Caracterizar la incidencia, fisiopatología y manejo del síndrome de Horner durante la anestesia neuroaxial. Métodos: Se realizó una

\section{Key words:}

Horner syndrome, neuraxial anesthesia, obstetric anesthesia

Palabras clave:

Síndrome de Horner, anestesia neuroaxial, anestesia obstétrica

Médico General, Universidad de los Andes.

Residente de Oftalmología, Universidad El Bosque. Bogotá, Colombia.

Fecha de recepción: 26 de junio de 2019

Fecha de aceptación: 15 de julio de 2019

ORCID

https://orcid.org/0000-0003-3231-893X

Correspondencia:

Anamaria Leguizamon Stevenson

Email: anamarialeguizamon07@gmail.com 
revisión de literatura mediante la búsqueda de artículos relacionados con el tema en PubMed y Web of Science. Resultados: La incidencia del síndrome de Horner tras bloqueo neuroaxial es muy variable siendo mayor en casos de cesárea que durante trabajo de parto ( $4 \%$ vs $0,4 \%-1,3 \%$ ). Factores que promueven el esparcimiento cefálico del anestésico, la sensibilidad neuronal incrementada, los cambios fisiológicos y anatómicos inducidos por el embarazo, y en algunos casos el mal posicionamiento del catéter, explicarían esta asociación. La mayoría de veces se trata de un fenómeno benigno el cual se resuelve espontáneamente en menos de 4 horas; no obstante, se debe monitorizar la aparición de inestabilidad hemodinámica o sintomatología atípica que obliguen a ampliar los estudios diagnósticos y/o interrumpir el anestésico.

\section{Introducción}

E I síndrome de Horner es una complicación rara reportada de la anestesia neuroaxial. Los signos neurooftalmológicos que implican el síndrome de Horner (miosis, ptosis, anhidrosis) no solo causan ansiedad entre las pacientes y personal médico, sino que también pueden anunciar el inicio de hipotensión materna[1]. No obstante, la mayoría de veces se trata de un fenómeno benigno y la presencia del síndrome de Horner no debería llevar a la interrupción del bloqueo neuroaxial, pero sí a una monitorización de presión arterial y de bienestar materno-fetal continua[2].

Actualmente, el bloqueo neuroaxial durante el trabajo de parto, es una práctica anestésica frecuente (en más del $50 \%$ de los partos de instituciones en Estados Unidos)[3] y los reportes del síndrome de Horner como complicación durante anestesia epidural van en aumento. Por consiguiente, el propósito de esta revisión de tema es informar a los pacientes y médicos (anestesiólogos, neurólogos, oftalmólogos) sobre la incidencia, fisiopatología y manejo del síndrome de Horner durante la anestesia epidural.

\section{Metodología}

Se realizó una revisión de la literatura en marzo de 2019 a través de búsqueda de artículos en PubMed (MEDLINE) y Web of Science utilizando una estrategia de búsqueda con términos Descriptores en ciencias de la Salud (DeCS) y Medical subject heading (MeSH). Se emplearon operadores boleanos AND y OR con la siguiente estructura: (Horner syndrome OR miosis OR ptosis OR anhydrosis OR autonomic nervous system diseases) AND (anaesthesia, epidural OR anaesthesia, spinal OR anaesthesia, caudal OR analgesia, obstetric OR anesthesia, peridural OR anesthesia, extradural) AND (pregnant OR obstetric OR labour OR cesarean section OR gravidity). No se utilizaron filtros durante la búsqueda. Se obtuvo 156 artículos en PubMed y 90 artículos en Web of Science. De estos se incluyeron 23 artículos tras revisar Título y Resumen. Adicionalmente, se revisaron las referencias bibliográficas de los artículos seleccionados. No se realizó la búsqueda en la literatura gris.

\section{Incidencia de la enfermedad}

En el mundo no hay datos epidemiológicos del síndrome de Horner aislado en adultos[4]. Los datos disponibles provienen de un estudio poblacional en Estados Unidos en población pediátrica. En éste la incidencia ajustada para edad y sexo fue de 1,42 por 100.000 pacientes menores de 19 años. Once de los 20 casos fueron congénitos y la prevalencia al nacimiento fue de 1 en 6.250 [95\% Cl 3.333 a 10.000] [5].

Por otro parte, la analgesia epidural-espinal combinada y epidural lumbar son comúnmente usadas para el alivio del dolor durante el parto debido a su efectividad y seguridad[6]. De hecho, la anestesia general durante la cesárea ha disminuido enormemente[7]. En un estudio prospectivo en Estados Unidos, en el que participaron 30 instituciones, se recolectaron durante 5 años datos de aproximadamente 257.000 pacientes a quienes se les administró anestesia neuroaxial o general. En éste se empleó anestesia neuroaxial en $76 \%$ de los partos vaginales y en $94,4 \%$ de las cesáreas en 5 años[8]. En Colombia, no hay datos epidemiológicos concretos disponibles, pero es importante resaltar que si bien el bloqueo neuroaxial es cada vez más frecuente en nuestro medio, solo las mujeres con planes de atención privada acceden a ella sin impedimentos[9].

La literatura reporta una frecuencia de complicaciones oftalmológicas y neurológicas de 1 por cada 
10.000 procedimientos de anestesia neuroaxial. Estos reportes generalmente solo incluyen déficits neurológicos y oculares persistentes y severos, probablemente excluyendo pacientes con síntomas transitorios o leves. Dentro de las complicaciones neurooftalmológicas se encuentra el síndrome de Horner[10]. Este síndrome se ha asociado a diversas técnicas de anestesia regional[11]. De hecho, el primer caso de síndrome de Horner como efecto secundario al uso de anestesia epidural lumbar fue reportado en 1972 por Kepes[12]. Se cree que es más común después del bloqueo neuroaxial en mujeres embarazadas y ciertamente se ha descrito con mayor frecuencia en gestantes tras anestesia epidural probablemente por cambios fisiológicos y anatómicos que acontecen durante la gravidez[11]. Al buscar en población no grávida se encuentra un estudio de 439 pacientes quienes reciben analgesia epidural torácica tras mastectomía, con una incidencia de síndrome de Horner similar (1,4\%) a la reportada en población obstétrica[1].

Ahora bien, la incidencia de Síndrome de Horner reportada en anestesia epidural durante trabajo de parto oscila entre $0,4 \%-1,3 \%$ y para cesárea hasta $4 \%[11]$. No obstante, dado el espectro de severidad de los síntomas de esta entidad, se cree que hay un subregistro epidemiológico de los casos pues la mayoría de pacientes son asintomáticas[13] o los cambios sutiles clínicos pueden haber sido ignorados durante su presentación. Por ejemplo, en un estudio prospectivo se demostró una incidencia de $75 \%$ de síndrome de Horner en embarazadas que reciben anestesia epidural[14] cuando específicamente se exploraba por cambios pupilares u otras manifestaciones de bloqueo simpático cervical[15]. En este mismo estudio la anestesia caudal se asoció con una incidencia mayor de síndrome de Horner, reportada como de 55\%[1]. Particularmente durante la búsqueda no se encontraron reportes de síndrome de Horner asociado a anestesia obstétrica espinal.

\section{Fisiopatología y abordaje de la enfermedad}

El síndrome de Horner se origina de un bloqueo de las fibras simpáticas altas (C8-T1) a nivel central o periférico ${ }^{16}$. La mayoría de veces es unilateral y se adquiere por una patología subyacente; sin embargo, su etiología puede ser congénita o iatrogénica[14]. Clínicamente se presenta con ptosis y miosis por parálisis del músculo de Müller y dilatador del iris respectivamente, enoftalmos por efecto visual de la ptosis, anhidrosis por denervación de las fibras simpáticas y enrojecimiento facial por pérdida del control vasomotor[16].
Varias teorías se han propuesto como causa del síndrome de Horner asociado a anestesia neuroaxial en el embarazo. No obstante, el mecanismo exacto no es conocido y muchos factores están involucra$\operatorname{dos}[13]$ :

1. Factores que promueven el esparcimiento cefálico del anestésico local: hallazgos fibroscopios han demostrado que existen cambios inducidos por la gestación en el espacio epidural que pueden afectar la propagación de la analgesia epidural[17]. Debido a un aumento de la presión abdominal y la congestión venosa epidural, el espacio que rodea la duramadre (epidural) se reduce[18]. La sangre se desvía de la vena cava inferior a través de los plexos venosos epidurales debido a la compresión del útero grávido, reduciendo aún más este espacio epidural[19]. Además, las presiones dentro de este espacio pueden elevarse durante las contracciones uterinas y las maniobras de valsalva lo que facilita la propagación del anestésico[10] y explica la frecuente aparición del síndrome hasta la etapa final de pujo[13]. De esta manera, la inyección de anestésico en el espacio epidural llegará a un nivel vertebral más alto en mujeres embarazadas[1] que en no grávidas.

2. Sensibilidad neuronal incrementada al anestésico local: se ha sugerido que los niveles altos de progesterona durante el embarazo pueden incrementar la sensibilidad de las fibras nerviosas[1]. En efecto la ocurrencia en muchos casos de síndrome de Horner en ausencia de bloqueo sensorial puede explicarse por esta alta sensibilidad de las fibras simpáticas (fibras tipo B)[16],[20] a los anestésicos locales y el hecho que el bloqueo simpático parece ser más cefálico que el sensorial[21].

3. Variaciones anatómicas: se han encontrado variaciones anatómicas que no solo pueden predisponer al síndrome de Horner, sino también determinar la unilateralidad del bloqueo. Entre estas se encuentran: la plica mediana dorsalis que tabica el espacio epidural[16], la existencia de fibras oculosimpáticas que se originan de niveles tan bajos como T4 y la variabilidad en el origen de la inervación simpática entre los lados derecho e izquierdo del mismo individuo[22].

4. Inadecuada posición del catéter: el espacio subdural se encuentra entre la aracnoides y la duramadre. Se cree que la canulación subdural ocurre en el $0,8 \%$ de todos los intentos de analgesia epidural y a menudo no se reconoce clínicamente[13].

En la mayoría de casos el curso de la enfermedad es benigno y se resuelve espontáneamente. El tiempo promedio para la resolución de los síntomas 
es de 215 minutos con un rango que oscila entre pocos minutos hasta 24 horas dependiendo del agente usado[13]. Sin embargo, es indispensable considerar otras condiciones asociadas al síndrome de Horner durante la anestesia neuroaxial obstetrica dado el efecto inhibitorio en la cadena simpática alta inducida por la dispersión cefálica del medicamento. Dentro de estas se encuentra: parálisis de nervios craneanos, dificultad respiratoria, hipotensión e inestabilidad hemodinámica[2]; estas últimas no solo representan las consecuencias más serias y potencialmente fatales del bloqueo espinal extenso producido por el anestésico, sino que pueden ser las manifestaciones iniciales de una falla del sistema nervioso autónomo. Por esto, siempre se debe monitorizar la aparición de complicaciones autonómicas postanestesia debido a la posibilidad de un bloqueo simpático alto[1].

Así pues, durante el abordaje se recomienda:

- Notificar a los profesionales de la salud a cargo de la paciente.

- Evaluar vía aérea, respiración y circulación (A-BC) buscando especialmente hipotensión materna y bienestar fetal. Adicionalmente, valorar el nivel sensitivo según dermatomas y valorar nervios motores[1].

- Evaluar el correcto posicionamiento del catéter. La mayoría de veces el diagnóstico de una mala localización será clínico (bloqueo sensitivo extenso con o sin bloqueo motor en caso de anestesia subdural)[13]. Aunque no hay guías claras para el manejo de un inadecuado posicionamiento, varios autores sugieren que ante cualquier duda se debe retirar y reposicionar el catéter[23],[24].

- Tomar una decisión individualizada de si continuar o detener la infusión de anestésico local (si aplica) con el fin de evitar hipotensión o dificultad respiratoria materna y asegurar el bienestar fetal[1]. En el contexto obstétrico, generalmente benigno, el diagnóstico extenso no está indicado, aunque se debe tener en cuenta la posibilidad de que se produzcan complicaciones fatales, siempre y cuando el paciente sea asintomático y hemodinámicamente estable, la epidural se puede continuar de forma segura[13].

\section{Discusión}

El Síndrome de Horner tras bloqueo neuroaxial obstétrico es una entidad poco nombrada en la literatura médica; aunque se encuentran casos reportados en revistas de obstetricia y anestesiología, en la literatura de oftalmología se encuentra un solo artículo publicado en 1995[12].

La mayoría de casos reportados de síndrome de Horner son en mujeres embarazadas probablemente por los cambios anatómicos producidos por el estado de gravidez. Varios autores concuerdan que la mayor dispersión cefálica del anestésico, causada por los cambios anatómicos durante la gestación, explica la interrupción simpática[25].

Cuando se presenta una paciente embarazada con alguna manifestación neurooftalmológica tras recibir bloqueo neuroaxial es importante sospechar un síndrome de Horner y plantearlo como diagnóstico diferencial. A menudo esta entidad pasa desapercibida por la escasa o variable sintomatología y resolución espontánea[16], lo cual no solo refleja su variable incidencia, sino invita a realizar su búsqueda activa en el contexto adecuado.

Existen diferentes opiniones en cuanto a si interrumpir o no el anestésico. Para algunos autores, cuando se sospecha bloqueo simpático alto debe suspenderse el anestésico prontamente[13]. Para otros, la continuación de la anestesia es posible; lo más importante será la vigilancia estricta, y en caso de sospechar paro respiratorio será prudente interrumpir el anestésico y elegir otra alternativa como analgesia[14]. De esta manera, el manejo es totalmente individual. Por ejemplo, puede presentarse una paciente en la que el síndrome de Horner sea el primer signo de un bloqueo extenso, con alta probabilidad de inestabilidad hemodinámica y compromiso materno fetal[26]; en esta ocasión es fundamental la observación, vigilancia y estudio extenso hasta la desaparición de los síntomas, pudiéndose optar por la interrupción de la perfusión del anestésico[25] según criterio médico.

En conclusión, el síndrome de Horner puede presentarse como una complicación tras anestesia neuroaxial en mujeres gestantes. El reconocimiento de este cuadro por parte de los profesionales de la salud es fundamental para asegurar su correcto abordaje; si bien la mayoría de veces es de buen pronóstico y resuelve espontáneamente, se debe estar atento a la aparición de anormalidades hemodinámicas o neurológicas que requieran una evaluación diagnóstica ampliada, mayor monitorización materno fetal o la interrupción del anestésico. 


\section{Referencias}

1. Chambers DJ, Bhatia K. Horner's syndrome following obstetric neuraxial blockade - a systematic review of the literature. Int J Obstet Anesth. 2018 Aug;35:75-87. https://doi. org/10.1016/j.ijoa.2018.03.005 PMID:29657082

2. Jadon A. Horner's syndrome and weakness of upper limb after epidural anaesthesia for caesarean section. Indian J Anaesth. 2014 Jul;58(4):464-6. https://doi.org/10.4103/00195049.139012 PMID:25197119

3. Vincent RD Jr, Chestnut DH. Epidural analgesia during labor. Am Fam Physician. 1998 Nov;58(8):1785-92. PMID:9835854

4. Proctor C, Chavis PS. Horner syndrome. J Neuroophthalmol. 2013 Mar;33(1):88-9. https://doi.org/10.1097/ WNO.0b013e318270ab95 PMID:23222307

5. Smith SJ, Diehl N, Leavitt JA, Mohney BG. Incidence of pediatric Horner syndrome and the risk of neuroblastoma: a population-based study. Arch Ophthalmol. 2010 Mar;128(3):324-9. https://doi.org/10.1001/ archophthalmol.2010.6 PMID:20212203

6. Pan PH, Bogard TD, Owen MD. Incidence and characteristics of failures in obstetric neuraxial analgesia and anesthesia: a retrospective analysis of 19,259 deliveries. Int J Obstet Anesth. 2004 Oct;13(4):227-33. https://doi. org/10.1016/j.ijoa.2004.04.008 PMID:15477051

7. Páez JJ, Navarro JR. Anestesia regional versus general para parto por cesárea. Rev Colomb Anestesiol. 2012 Aug;40(3):203-6. https://doi.org/10.1016/j. rca.2012.05.008.
8. D'Angelo R, Smiley RM, Riley ET, Segal S. Serious complications related to obstetric anesthesia: the serious complication repository project of the Society for Obstetric Anesthesia and Perinatology. Anesthesiology. 2014 Jun;120(6):1505-12. https://doi.org/10.1097/ ALN.0000000000000253 PMID:24845921

9. Herrera PJ, Medina PA. Los problemas de la analgesia obstétrica. Rev Colomb Anestesiol. 2014 Jan;42(1):37-9. https://doi. org/10.1016/j.rca.2013.09.006.

10. Biousse V, Guevara RA, Newman NJ. Transient Horner's syndrome after lumbar epidural anesthesia. Neurology. 1998 Nov;51(5):1473-5. https://doi. org/10.1212MNL.51.5.1473 PMID:9818887

11. Jiménez $F$, Borràs $R$, Mailan J, Molins G, Vacas Y. Síndrome de Horner y bloqueo del plexo braquial tras anestesia epidural para trabajo de parto y cesárea. Rev Esp Anestesiol Reanim. 2011;58:54-6. http:// dx.doi.org/10.1016/s00349356(11)70698-3

12. Goel S, Burkat CN. Unusual case of persistent Horner's syndrome following epidural anaesthesia and caesarean section. Indian J Ophthalmol. 2011 SepOct;59(5):389-91. https://doi. org/10.4103/0301-4738.83620 PMID:21836349

13. Barbara R, Tome R, Barua A, Barbara A, Gaitini L, Odeh M, et al. Transient Horner syndrome following epidural anesthesia for labor: case report and review of the literature. Obstet Gynecol Surv. 2011 Feb;66(2):114-9. https://doi.org/10.1097/ OGX.0b013e31821d6e5c PMID:21592418

14. Sharma R, Chatterjee J, Edmonds K. Horner's syndrome with epidural anaesthesia. BMJ Case Rep.

2010 Aug;2010 aug23

1:bcr0120102698. https://doi. org/10.1136/bcr.01.2010.2698 PMID:22767365

15. Thomas DV. Letter: horner's syndrome following obstetric extradural block. Br J Anaesth. 1976 Jun;48(6):611. https:// doi.org/10.1093/bja/48.6.611 PMID:952697

16. Avellanosa J, Vera J, Morillas P, Gredilla E, Gilsanz F. Síndrome de Horner y bloqueo del plexo braquial ipsilateral en un caso de analgesia epidural para el trabajo del parto. Rev Soc Esp Dolor. 2006;13(7):481-4.

17. Igarashi $T$, Hirabayashi $Y$, Shimizu R, Saitoh K, Fukuda H, Suzuki $\mathrm{H}$. The fiberscopic findings of the epidural space in pregnant women. Anesthesiology. 2000 Jun;92(6):1631-6. https:// doi.org/10.1097/00000542200006000-00021 PMID:10839913

18. Lynch JH, Keneally RJ, Hustead TR. Horner's syndrome and trigeminal nerve palsy following epidural analgesia for labor. J Am Board Fam Med. 2006 SepOct;19(5):521-3. https://doi. org/10.3122/jabfm.19.5.521 PMID:16951303

19. Sprung J, Haddox JD, MaitraD'Cruze AM. Horner's syndrome and trigeminal nerve palsy following epidural anaesthesia for obstetrics. Can J Anaesth. 1991 Sep;38(6):767-71. https:// doi.org/10.1007/BF03008457 PMID:1914062

20. Heavner JE, de Jong RH. Lidocaine blocking concentrations for B- and C-nerve fibers. Anesthesiology. 1974 Mar;40(3):228-33. https:// doi.org/10.1097/00000542197403000-00004 PMID:4814244

21. Theodosiadis PD, Grosomanidis VO, Gkoutzioulis FV, Tzafettas 
JM. A case of unilateral Horner's syndrome after combined spinal epidural anesthesia with ropivacaine $10 \mathrm{mg} / \mathrm{mL}$ for cesarean section. Int J Obstet Anesth. 2006 Jan;15(1):68-70. https://doi. org/10.1016/j.ijoa.2005.05.010 PMID:16325391

22. Ray BS, Hinsey JC, Geohegan WA. Observations On The Distribution Of The Sympathetic Nerves To The Pupil And Upper Extremity As Determined By Stimulation Of The Anterior Roots In Man. Ann Surg. 1943 Oct;118(4):647-55. https:// doi.org/10.1097/00000658-
194310000-00013

PMID: 17858298

23. Chen SH, Chiueh HY, Hung CT, Tsai SC, Wong SY. Extensive sensory block caused by accidental subdural catheterization during epidural labor analgesia. Chang Gung Med J. 2006 Nov-Dec;29(6):607-11. PMID:17302226

24. Rowbottom SJ. Continuous subdural block. Br J Anaesth. 1993 Oct;71(4):607. https:// doi.org/10.1093/bja/71.4.607-a PMID:8204147

25. Rodríguez-Sánchez $E$, Vadillo JM, Herrera-Calo P, Marenco de la Fuente ML. Marenco de la Fuente, María Luisa. Síndrome de Horner tras analgesia epidural para el parto. Informe de 3 casos. Rev Colomb Anestesiol. 2016;44(2):170-3. https://doi.org/10.1016/j. rca.2016.01.004.

26. Narouze SN, Basali A, Mandel $M$, Tetzlaff JE. Horner's syndrome and trigeminal nerve palsy after lumbar epidural analgesia for labor and delivery. J Clin Anesth. 2002 Nov;14(7):5324. https://doi.org/10.1016/ S0952-8180(02)00406-3 PMID:12477590 